\title{
Comments on "Incidence of cancer among licensed commercial pilots flying North Atlantic routes"
}

\author{
S. M. J. Mortazavi
}

\begin{abstract}
Gudmundsdottir et al. in their paper entitled "Incidence of cancer among licensed commercial pilots flying North Atlantic routes" published in Environmental Health have evaluated the effects of exposure to higher levels of cosmic radiation on cancer incidence in the pilots of commercial flights. Despite its remarkable strengths, the paper authored by Gudmundsdottir et al. has some shortcomings. The shortcomings of this paper such as not determining the shape of dose-response relationship for radiation-induced cancers, limitations in flight dose calculations, the weaknesses of CARI- 6 as the program used by Gudmundsdottir et al. to estimate the effective dose of galactic cosmic rays, and the problems associated with unpredictable nature of the magnitude and duration of solar particle events are discussed.
\end{abstract}

Keywords: Cancer, Pilots, Commercial flights, Cosmic radiation

\section{Background}

Pilots and aircrew are occupationally exposed to higher levels of cosmic radiation compared to our exposures on Earth [1] that is caused by decreased shielding role of the Earth's atmosphere and the Earth's magnetic field at flight altitudes [2]. Higher incidence of melanoma in pilots and cabin crew compared to the general population is previously reported [3]. This commentary is regarding the article by Gudmundsdottir et al. entitled "Incidence of cancer among licensed commercial pilots flying North Atlantic routes" published in Environmental Health [4]. The authors have tried to evaluate the effects of exposure to higher levels of cosmic radiation on cancer incidence in the pilots of commercial flights. The paper authored by Gudmundsdottir et al. has some shortcomings. These shortcomings and their possible effect on the validity of the findings of Gudmundsdottir et al. are discussed in detail in the following section.

\section{Comments}

The first shortcoming of this study is due to not determining the shape of dose-response relationship for

\footnotetext{
* Correspondence: S.M.Javad.Mortazavi@fccc.edu

Diagnostic Imaging Center, Fox Chase Cancer Center, 333 Cottman Avenue, Philadelphia, PA 19111, USA
}

radiation-induced cancers while the authors were aware of the importance of the exposure level and cumulative radiation dose "The RRs for all cancers, prostate cancer, melanoma, BCC, and BCC of trunk are highest in the highest exposure categories of cumulative radiation dose, and cumulative radiation dose sustained before the age of 40 years as compared with corresponding categories of duration of employment". It is worth noting that the shape of the dose-response curve for cancer after exposure to low doses of ionizing radiation has been a highly controversial issue for the past several decades [5]. Substantial data now indicates that in contrast to the predictions of the linear non-threshold (LNT) model; i.e. any dose of radiation, no matter how small, increases the risk of cancer [6], ionizing radiation induced-cancers may have specific thresholds of radiation dose.

Another shortcoming of this paper comes from the limitations in flight dose calculations. It is known that the factors determine the exposures of air crew are not limited to flight altitude, latitude and duration. For example, the solar activity plays a key role in this issue. Although the biological effects of the exposure of aircrew to galactic cosmic ray (GCR) are usually much larger than those of the occasional solar particle events (SPE), an intense SPE can lead to a 
significant rise in dose $[7,8]$. While applications such as CARI-6 can be used to estimate the effective dose of GCRs, they cannot be used for estimating the effective dose received from SPEs. Despite intense SPEs are rare, the magnitude and duration of SPEs are currently unpredictable and hence they cannot be entirely ignored.

Furthermore CARI-6 unlike its updated version CARI-7A, cannot directly include heavy ion transport [9] and the effective doses calculated with CARI-7 for some flights show a significant difference (ranged from $-9.2 \%$ to $+23 \%$ ) with those calculated with CARI-6 [10].

\section{Conclusion}

Despite its remarkable strengths, the paper authored by Gudmundsdottir et al. has shortcomings such as not determining the shape of dose-response relationship for radiation-induced cancers, methodological problems in flight dose calculations and the limitations of CARI-6 compared to its updated version CARI-7A. These shortcomings raise questions over the validity of the findings derived.

\section{Response to comments on "Incidence of cancer among licensed pilots flying North Atlantic routes"} Eva Maria Gudmundsdottir ${ }^{1,2,3}$, Jon Hrafnkelsson ${ }^{1,2,3}$ and Vilhjalmur Rafnsson 1,2,3

${ }^{1}$ Faculty of Medicine, University of Iceland, Reykjavik, Iceland

${ }^{2}$ Department of Oncology, Landspitali University Hospital, Reykjavik, Iceland

${ }^{3}$ Department of Preventive Medicine, Faculty of Medicine, University of Iceland, Reykjavik, Iceland

\begin{abstract}
Our study "Incidence of cancer among licensed commercial pilots flying North Atlantic routes" (4) has been commented on. We evaluated incidence of several cancer types and locations in association with cosmic radiation, and observed a positive exposure-response relation for incidence of the radiation-related cancer, basal cell carcinoma of the skin (BCC), with the four exposure metrics: employment years, cumulative air hours, cumulative radiation dose, and cumulative radiation dose sustained up to 40 years of age. The strongest relations were observed with total cumulative radiation dose and cumulative radiation dose sustained up to the age of 40 years.
\end{abstract}

Keywords: Basal cell carcinoma of skin, cosmic ionizing radiation, exposure-response relation

\section{Background}

We would like to thank dr. Mortazavi for his comment regarding our study on licensed commercial pilots (4), and at the same time we would like to clarify that we evaluated incidence of several cancer types and locations in association with gradient of cosmic radiation, and in spite of the smallness of the cohort, observed a positive exposure-response relation for incidence of the radiation-related cancer, basal cell carcinoma of the skin (BCC), with all four exposure metrics, and the strongest relation were observed with total cumulative radiation dose and cumulative radiation dose sustained up to the age of 40 years.

\section{Comments}

We agree with dr. Mortazavi that the use of the recently updated and improved version of the computer software CARI-7 (9) may have improved the estimation of the effective dose of ionizing radiation, and certainly we will consider that in future studies. We also agree with dr. Mortazavi that the possible biological effects of the exposure of aircrew to galactic cosmic ray are usually considered much larger than those of the solar particle events. As dr. Mortazavi says, the occurrences of solar particle events are rare, however, may be of especially short duration, and in order to take these into account in the exposure estimation in a cohort of pilots, it is necessary to have knowledge of whether each individual pilot has been flying at the time of the event, and our data do not have that precision. In our estimate of exposure metrics we had access to and used airline reports on annual number of block hours (converted to air hours), per year, per aircraft type, and per individual pilot, and that condition is of clear advantage compared with the use of retrospective self-reported work history, as has been the case in some aircrew studies.

We do not consider it appropriate, on basis of our study, to go into in-depth discussion on the predictions of the linear non-threshold model, i.e. that any small dose of radiation increase the risk of cancer, or whether ionizing radiation induced-cancers may have specific thresholds of radiation dose, but dr. Mortazavi mentions these in his commentary. In our article we frequently complained that one of the limitation of our study (4), is that the cohort is small, and the cases are not so numerous, leaving us with lack of power for complicated investigation or description of the shape of the exposureresponse relation, and one of our conclusion (4) is that this relation needs to be clarified more exactly in future 
studies. We analysed whether the risk of cancers did become greater with gradually higher levels of exposure to cosmic radiation, where licensed pilots not employed at the airline company belonged to the lowest exposure category, and licensed pilots employed at the airline company were divided into two exposure categories, lower and higher, by the median of sustained ionizing radiation dose in milliSievert $(\mathrm{mSv})$ in generalized linear model (4). In these calculations for the altogether 31 cases of BCC the relative risk (RR) became higher with higher exposure categories and the trends were statistically significant. However, according to the discussion above, we are not able to conclude on the possibility of threshold level. So in our analyses of cancer incidence in relation to the exposures we were not only evaluating "effects of exposure to higher levels of cosmic radiation on cancer incidence", as maintained by dr. Mortazavi, but to a gradient of exposure categories (4).

In our calculations, the association of RR for BCC with ionizing radiation dose in $\mathrm{mSv}$, we introduced the exposure metrics as continuous variables in the Poisson regression. In that analysis the RR was 1.0215 (95\% confidence interval (CI) 1.0082 to 1.0349) for all BCC, and the RR was 1.0369 (95\% CI 1.0133 to 1.0649) for BCC of trunk in association with $1 \mathrm{mSv}$ increase of total cumulative radiation dose; the RR was 1.0001 (95\% CI 1.0000 to 1.0002 ) for all BCC, and the RR was 1.0001 (95\% CI 1.0000 to 1.0002) for BCC of trunk in association with $1 \mathrm{mSv}$ increase of cumulative radiation dose sustained before age of 40 years. These figures are not describing the exact shape of the exposure-response relation, however, shown here for more detailed information.

\section{Conclusions}

In summary the most important result of our study is the strong positive exposure-response relation observed for incidence of all BCC, and BCC of trunk with the estimated total cumulative ionizing radiation dose in $\mathrm{mSv}$, and cumulative ionizing radiation dose in $\mathrm{mSv}$ sustained up to 40 years of age. The strength of the study, as compared to previous studies on aircrews, is the use of airline reports for the calculation of the individual ionizing radiation dose, and the record linkage of the personal identifier with the comprehensive cancer registry when obtaining accurate information on the outcome, the cancer incidence. The study is the first to show that the radiation-related cancer $\mathrm{BCC}$, which is not a deadly cancer, is associated with the exposure to cosmic radiation, thus the BCC among the pilots may be attributed to the cosmic radiation.

\section{Abbreviations}

GCR: galactic cosmic rays; LNT: linear non-threshold; SPE: solar particle events
Funding

Not applicable.

Availability of data and materials

No additional data available.

Author's contributions

The author actively reviewed and criticized the original paper, drafted and finalized the commentary.

Ethics approval and consent to participate

Not applicable.

Consent for publication

Not applicable.

Competing interests

The author declares that he has no competing interests.

\section{Publisher's Note}

Springer Nature remains neutral with regard to jurisdictional claims in published maps and institutional affiliations.

Received: 8 September 2017 Accepted: 1 November 2017 Published online: 17 November 2017

References

1. Alves MC, Galeano DC, Santos WS, Lee C, Bolch WE, Hunt JG, da Silva AX, Carvalho $\mathrm{AB}$. Comparison of the effective dose rate to aircrew members using hybrid computational phantoms in standing and sitting postures. J Radiol Prot. 2016;36(4):885-901.

2. Bagshaw M. Cosmic radiation in commercial aviation. Travel Med Infect Dis. 2008;6(3):125-7.

3. Shantha E, Lewis C, Nghiem P. Why do airline pilots and flight crews have an increased incidence of melanoma? JAMA Oncol. 2015;1 (6):829-30.

4. Gudmundsdottir EM, Hrafnkelsson J, Rafnsson V. Incidence of cancer among licenced commercial pilots flying North Atlantic routes. Environ Health. 2017;16(1):86

5. Scott BR, Walker DM, Tesfaigzi Y, Schöllnberger H, Walker V. Mechanistic basis for nonlinear dose-response relationships for low-dose radiationinduced stochastic effects. Nonlinearity Biol Toxicol Med. 2003;1(1):93-122.

6. de Toledo SM, Azzam El. Adaptive and bystander responses in human and rodent cell cultures exposed to low level ionizing radiation: the impact of linear energy transfer. Dose-Response. 2006;4(4):291-301.

7. Mortazavi SMJ, Cameron JR, Niroomand-rad A. Adaptive response studies may help choose astronauts for long-term space travel. Adv Space Res. 2003;31(6):1543-51.

8. Mortazavi SMJ, Cameron JR, Niroomand-Rad A. The life saving role of radioadaptive responses in long-term interplanetary space journeys. Int Congr Ser. 2005;1276:266-7.

9. Copeland K. CARI-7A: development and validation. Radiat Prot Dosim. 2017; 175(4):419-31.

10. U.S. Department of Transportation FAA: in-flight radiation exposure. Advisory Circular. 2014:120-61B.

Submit your next manuscript to BioMed Central and we will help you at every step:

- We accept pre-submission inquiries

- Our selector tool helps you to find the most relevant journal

- We provide round the clock customer support

- Convenient online submission

- Thorough peer review

- Inclusion in PubMed and all major indexing services

- Maximum visibility for your research

Submit your manuscript at www.biomedcentral.com/submit 